\title{
Symptoms of Rapid Eye Movement Sleep Behavior Disorder are Associated with Cholinergic Denervation in Parkinson Disease
}

\author{
Vikas Kotagal, MD, ${ }^{1}$ Roger L. Albin, MD, ${ }^{1,2}$ Martijn L. T. M. Müller, PhD, ${ }^{3}$ \\ Robert A. Koeppe, PhD, ${ }^{3}$ Ronald D. Chervin, MD, MS, ${ }^{1}$ Kirk A. Frey, MD, PhD, ${ }^{1,3}$ \\ and Nicolaas I. Bohnen, MD, PhD ${ }^{1,2,3}$
}

Objective: Rapid eye movement sleep behavior disorder (RBD) is common in Parkinson disease (PD), but its relationship to the varied neurotransmitter deficits of PD and prognostic significance remain incompletely understood. RBD and cholinergic system degeneration are identified independently as risk factors for cognitive impairment in PD. We aimed to assess the association between cholinergic denervation and symptoms of RBD in PD patients without dementia.

Methods: Eighty subjects with PD without dementia (age, $64.6 \pm 7.0$ years; range, $50-82$ years; 60 males, 20 females; mean Montreal Cognitive Assessment Test [MoCA] score, $26.2 \pm 2.1$; range 21-30) underwent clinical assessment, neuropsychological testing, and $\left[{ }^{11} \mathrm{C}\right]$ methylpiperidyl propionate acetylcholinesterase and $\left[{ }^{11} \mathrm{C}\right]$ dihydrotetrabenazine (DTBZ) vesicular monoamine transporter type 2 positron emission tomography (PET) imaging. ${ }^{11}$ C3-Amino-4-(2-dimethylaminomethyl-phenylsulfaryl)-benzonitrile (DASB) serotonin transporter PET imaging was performed in a subset of 35 subjects. The presence of RBD symptoms was determined using the Mayo Sleep Questionnaire.

Results: Twenty-seven of 80 subjects (33.8\%) indicated a history of RBD symptoms. Subjects with and without RBD symptoms showed no significant differences in age, motor disease duration, MoCA, Unified Parkinson Disease Rating Scale motor scores, or striatal DTBZ binding. Subjects with RBD symptoms, in comparison to those without, exhibited decreased neocortical, limbic cortical, and thalamic cholinergic innervation $(0.0213 \pm 0.0018$ vs $0.0236 \pm$ $0.0022, t=4.55, p<0.0001 ; 0.0388 \pm 0.0029$ vs $0.0423 \pm 0.0058, t=2.85, p=0.0056 ; 0.0388 \pm 0.0025$ vs 0.0427 $\pm 0.0042, t=4.49, p<0.0001$, respectively). Brainstem and striatal DASB binding showed no significant differences between groups.

Interpretation: The presence of RBD symptoms in PD is associated with relative neocortical, limbic cortical, and thalamic cholinergic denervation although not with differential serotoninergic or nigrostriatal dopaminergic denervation. The presence of RBD symptoms may signal cholinergic system degeneration.

ANN NEUROL 2012;71:560-568

$\mathrm{R}$ apid eye movement (REM) sleep behavior disorder (RBD) is characterized by loss of normal atonia and oneiric behavior, including abnormal and sometimes violent motor manifestations, during REM sleep. ${ }^{1} \mathrm{RBD}$ is a common nonmotor comorbidity of Parkinson disease (PD) and may be associated with increased risk of cognitive impairment. ${ }^{2,3} \mathrm{PD}$ is a multisystem neurodegenera- tive syndrome, and cognitive impairment in PD is likely the result of neurodegeneration within several brain systems associated with multiple neurotransmitter deficiencies. $^{4,5}$ Striatal dopaminergic denervation probably contributes to cognitive impairments in PD, but deficits of other neurotransmitter systems, notably cholinergic pathways, are also important. In contrast to middle stage

View this article online at wileyonlinelibrary.com. DOI: 10.1002/ana.22691

Received Aug 10, 2011, and in revised form Oct 24, 2011. Accepted for publication Dec 2, 2011.

Address correspondence to Dr Vikas Kotagal, Department of Neurology, University of Michigan Medical School, 2301 Commonwealth Boulevard, Room 1013, Ann Arbor, Ml 48105-2945. E-mail: vikaskot@med.umich.edu

From the ${ }^{1}$ Department of Neurology, University of Michigan, ${ }^{2}$ Neurology Service and Geriatric Research, Education, and Clinic Center, Veterans Administration Ann Arbor Health System; and ${ }^{3}$ Department of Radiology, Division of Nuclear Medicine, University of Michigan, Ann Arbor, MI. 
(Braak 3 and 4) PD, where there is uniform and severe striatal dopaminergic denervation, ${ }^{6}$ convergent evidence from in vivo imaging and postmortem studies indicates that the magnitude of forebrain cholinergic deficits increases over the course of PD disease progression and correlates significantly with the presence of cognitive impairment in PD. ${ }^{5,7-10}$ Hilker et al, for example, did not observe differences in striatal fluorodopa uptake reductions between $\mathrm{PD}$ and $\mathrm{PD}$ with dementia (PDD) groups, but the PDD group had significantly reduced neocortical acetylcholinesterase (AChE) activity compared to PD subjects. ${ }^{9}$ Cholinergic system deficits appear to advance in parallel with the progression of cognitive impairments. ${ }^{5,7-10}$

As both RBD and cholinergic denervation are risk factors for cognitive impairment in PD, we aimed to investigate the potential association between RBD symptoms and cholinergic system integrity in neocortical, thalamic, and limbic cortical regions of PD subjects. We compared cholinergic system changes with the degree of nigrostriatal dopaminergic denervation in the same subjects.

Another important subcortical system that may be involved in the pathogenesis of RBD is the brainstem serotoninergic system. Rostral brainstem serotonergic nuclei innervate cortical and subcortical structures, including the striatum, via projections arising from the raphe complex. Serotoninergic raphe projections within the brainstem may also regulate the normal function of circuits involved in the pathophysiology of RBD. ${ }^{11}$ Serotoninergic raphe complex neurons degenerate in $\mathrm{PD}$, and loss of serotoninergic raphe neurons may be a relatively early event in the course of PD. ${ }^{11,12}$ For this reason, we also evaluated serotoninergic raphe nucleus neuron integrity in a subset of subjects studied.

\section{Patients and Methods}

\section{Subjects and Clinical Test Battery}

This cross-sectional study involved 80 subjects with PD (20 women and $60 \mathrm{men}$ ). All subjects met the UK Parkinson Disease Society Brain Bank Research Center clinical diagnostic criteria for PD. ${ }^{13}$ Diagnosis of PD was confirmed by the presence of nigrostriatal dopaminergic denervation on $\left[{ }^{11} \mathrm{C}\right]$ dihydrotetrabenazine (DTBZ) positron emission tomography (PET) imaging. Patients had mild to moderate severity of disease: 3 subjects in Hoehn-Yahr (H-Y) stage 1, 5 in H-Y stage 1.5, 20 in H-Y stage 2, 40 in H-Y stage 2.5 , and 12 in H-Y stage $3 .{ }^{14}$ The mean duration of disease was $6.0 \pm 3.9$ (standard deviation) years, with a range of 1 to 19 years. Subjects with Montreal Cognitive Assessment (MoCA) scores $<20$ were excluded. ${ }^{15}$ The mean MoCA score was $26.2 \pm 2.1$ (range, 21-30). The Movement Disorder Society-revised Unified Parkinson Disease Rating Scale (UPDRS) was performed in the practically defined off state. ${ }^{16}$ No patients were using anticholi- nergic or cholinesterase inhibitor drugs, and no subjects who underwent ${ }^{11} \mathrm{C} 3$-amino-4-(2-dimethylaminomethyl-phenylsulfaryl)-benzonitrile (DASB) imaging were using drugs that might modify serotonergic neurotransmission.

\section{Standard Protocol Approvals, Registrations, and Patient Consents}

The study was approved by the institutional review board of the University of Michigan. Written informed consent was obtained from all subjects.

\section{Mayo Sleep Questionnaire}

Symptoms of RBD were assessed using the informant-based response to question \#1 on the Mayo Sleep Questionnaire (MSQ): "Have you ever seen the patient appear to 'act out his/ her dreams' while sleeping?" ${ }^{\prime 7}$ Affirmative replies were confirmed by positive responses to subquestions 1d ("Has the patients told you about dreams of being chased, attacked, or that involve defending himself/herself?") and 1e ("If the patient told you about a dream, did the details of the dream match the movements made while sleeping?") of the MSQ administered to the subject's bed partner. ${ }^{17}$ Question \#1 of the MSQ was validated against polysomnography in a multicenter prospective cohort study of normal elderly subjects and patients with suspected neurodegenerative disease; the sensitivity was $98 \%$, and specificity was $74 \%{ }^{17}$

The International Restless Leg Scale (IRLS) ${ }^{18}$ and the Parkinson's Disease Sleep Scale ${ }^{19}$ were also administered. These scales have been used in PD subjects to assess for restless legs syndrome (RLS) and periodic limb movement disorder (PLMD) of sleep. ${ }^{20,21}$

\section{Neuropsychological Examination}

Early neuropsychological abnormalities in PD are thought to manifest predominantly with a frontal-subcortical cognitive syndrome. This includes difficulties with executive functioning, working memory, attention, set-shifting, and to a lesser extent, visuospatial difficulties. ${ }^{5}$ Neuropsychological tests were chosen to examine these specific cognitive domains following an approach as reported previously in PD. ${ }^{22}$ The California Verbal Learning Test assesses learning/encoding memory difficulties thought to be a feature of early cognitive impairment in PD. ${ }^{23,24}$ Executive function was assessed with the Wechsler Adult Intelligence Scale III Picture Arrangement, used in PD as a nontimed test of picture sequencing to make it independent from motor impairments. ${ }^{25,26}$ The Delis-Kaplan Executive Function System sorting and letter verbal fluency subtests ${ }^{27}$ are abnormal in PDD, improve with cholinergic therapy, ${ }^{28}$ and may reflect severe cholinergic denervation. The Stroop Color Word Interference test ${ }^{29}$ and a switching version of the Stroop 3 test in which subjects name the ink, unless the word is surrounded by a box, in which case, they read the word itself $(\text { Stroop } 4)^{30}$ were both administered. Speed of performance on Stroop 1 and 2 reflects attentional difficulties ${ }^{29,31}$; calculating the difference between Stroop 3 and 4 measures an additional demand on cognitive flexibility in PD and has been used in 
previous studies by our group. ${ }^{32}$ Visuospatial function was assessed using the Benton Judgment of Line Orientation test used previously in PD. ${ }^{33,34}$ Composite $z$ scores were calculated for these different cognitive domains (memory, executive, attention, and visuospatial functions) based on normative data. Global cognitive performance was calculated as the average $z$ score for the 4 cognitive domains. Subjects with evidence of dementia defined as a global cognitive $z$ score $<-2$ and impaired instrumental activities of daily living were not eligible for this study.

\section{Imaging Techniques}

DTBZ is a ligand binding to the type 2 vesicular monoamine transporter (VMAT2) present in monoaminergic presynaptic terminals. When measured in the striatum, where $>95 \%$ of VMAT2 binding sites are associated with dopaminergic terminals, ${ }^{35,36}$ VMAT2 binding is a useful method for estimating nigrostriatal dopaminergic denervation, particularly as its expression is not thought to be significantly altered by chronic dopaminergic therapy. ${ }^{37}$ Methylpiperidynl propionate (PMP) binds to synaptic AChE, which is recognized as a reliable marker for brain cholinergic pathways. ${ }^{38,39}$ PMP undergoes hydrolysis to a metabolite retained in brain, and tracer metabolite retention is a direct function of AChE activity, a surrogate for the amount of regional $\mathrm{AChE}$ and cholinergic terminal integrity. DASB binds selectively to the presynaptic serotonin transporter (SERT) and is recognized as an appropriate surrogate marker for serotoninergic terminal density. ${ }^{40}$

DTBZ, PMP, and DASB PET imaging was performed in 3-dimensional (3D) imaging mode using an ECAT HR+ tomograph (Siemens Molecular Imaging, Knoxville, TN), which acquires 63 transaxial slices (slice thickness, $2.4 \mathrm{~mm}$; intrinsic inplane resolution, $4.1 \mathrm{~mm}$ full-width at half maximum over a $15.2 \mathrm{~cm}$ axial field of view). A NeuroShield (Scanwell Systems, Montreal, Canada) head-holder/shielding unit was attached to the patient's bed to reduce the contribution of detected photon events originating from the body outside the scanner field of view. ${ }^{41}$ Prior to radioligand injections, a 5-minute transmission scan was acquired using rotating ${ }^{68} \mathrm{Ge}$ rods for attenuation correction of emission data using the standard vendor-supplied segmentation and reprojection routines. All subjects were studied supine, with eyes and ears unoccluded, resting quietly in a dimly lit room.

\section{DTBZ PET Imaging}

No-carrier-added (+)-[ $\left[{ }^{11} \mathrm{C}\right] \mathrm{DTBZ}(250-1,000 \mathrm{Ci} / \mathrm{mmol}$ at the time of injection) was prepared as reported previously. ${ }^{42}$ Dynamic PET scanning was performed for 60 minutes immediately following a bolus injection of $55 \% 555 \mathrm{MBq}(15 \mathrm{mCi})(+)-$ $\left[{ }^{11} \mathrm{C}\right] \mathrm{DTBZ}$ (containing $<50 \mu \mathrm{g}$ of cold DTBZ mass) over the first 15 to 30 seconds of the study, whereas the remaining $45 \%$ of the dose was continuously infused over the next 60 minutes, resulting in stable arterial tracer levels and equilibrium with brain tracer levels after 30 minutes. ${ }^{43}$ A series of 15 scan frames over 60 minutes was obtained as following: $4 \times 30$ seconds; $3 \times 1$ minute; $2 \times 2.5$ minutes; $2 \times 5$ minutes; and $4 \times 10$ minutes.

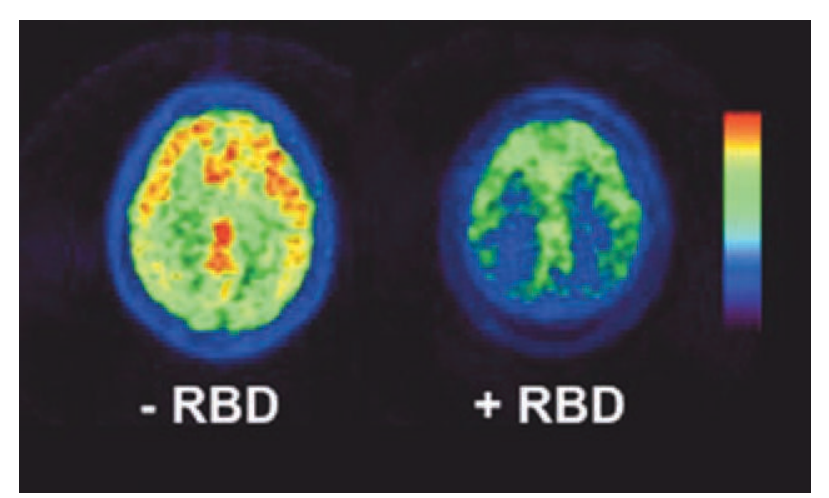

FIGURE: Neocortical $\left[{ }^{11} \mathrm{C}\right]$ methylpiperidyl propionate positron emission tomography signal in 2 subjects, 1 with (right) and another without (left) symptoms of rapid eye movement sleep behavior disorder (RBD). [Color figure can be viewed in the online issue, which is available at www.annalsofneurology.org]

\section{PMP PET Imaging}

$\left[{ }^{11} \mathrm{C}\right] \mathrm{PMP}$ was prepared in high radiochemical purity $(>95 \%)$ by $\mathrm{N}-\left[{ }^{11} \mathrm{C}\right]$ methylation of piperidin-4-yl propionate using a previously described method. ${ }^{44}$ Dynamic PET scanning was performed for 70 minutes immediately following a bolus intravenous injection of $666 \mathrm{MBq}(18 \mathrm{mCi})$ of $\left[{ }^{11} \mathrm{C}\right] \mathrm{PMP}$. The dose contained $<200 \mu \mathrm{g}$ cold PMP mass. Emission data were collected in 16 sequential emission scans (the DTBZ protocol plus an addition 10-minute frame).

\section{DASB PET Imaging}

The DASB PET studies were acquired as 17 sequential emission scans (the DTBZ protocol plus 2 additional 10-minute frames) in 35 subjects. Radiotracer was administered as a bolus (666mBq of $18 \mathrm{mCi}{ }^{11} \mathrm{CDASB}$ containing $<8 \mu \mathrm{g}$ of cold DASB) plus constant infusion using $70 \%$ as a slow bolus over 30 seconds, followed by constant infusion of the remaining $30 \%$ over the 80 -minute study duration.

\section{Magnetic Resonance Imaging}

All subjects underwent brain magnetic resonance imaging on a 3T Philips Achieva system (Philips, Best, the Netherlands) utilizing an 8-channel head coil and the ISOVOX exam card protocol primarily designed to yield isotropic spatial resolution. A standard T1-weighted series of a 3D inversion recovery-prepared turbo field echo was performed in the sagittal plane using repetition time/echo time/inversion time, 9.8/4.6/1,041 milliseconds; turbo factor, 200; single average; field of view, $240 \times$ $200 \times 160 \mathrm{~mm}$; acquired matrix, $240 \times 200$. One hundred sixty slices were reconstructed to $1 \mathrm{~mm}$ isotropic resolution. This sequence maximizes contrast among gray matter, white matter, and cerebrospinal fluid and provides high-resolution delineation of cortical and subcortical structures.

\section{Data Analysis}

Interactive Data Language image analysis software (Research Systems, Boulder, CO) was used to manually trace volumes of interest (VOIs) on magnetic resonance images to include the thalamus, limbic cortex (hippocampus and amygdala), cerebellar cortex, caudate nucleus, and putamen of each hemisphere. Total 


\section{TABLE 1: Demographic and Clinical Information in Patients with and without Symptoms of RBD}

\begin{tabular}{|c|c|c|c|}
\hline Characteristic & $\begin{array}{l}\text { PD Subjects with } \\
\text { RBD Symptoms, } \\
\mathbf{n}=27, \text { Mean } \pm \text { SD }\end{array}$ & $\begin{array}{l}\text { PD Subjects without } \\
\text { RBD Symptoms, } \\
\mathbf{n}=53, \text { Mean } \pm \text { SD }\end{array}$ & Statistical Significance \\
\hline Age & $63.4 \pm 6.7$ & $65.3 \pm 7.1$ & $t=1.12, p=0.26$ \\
\hline Gender & F $2 / \mathrm{M} 25$ & F 18/ M 35 & $\chi^{2}=6.7, p=0.009$ \\
\hline Duration of motor disease, yr & $6.4 \pm 3.7$ & $5.8 \pm 4.0$ & $t=-0.63, p=0.524$ \\
\hline MoCA score & $26.0 \pm 2.3$ & $26.3 \pm 2.0$ & $t=0.60, p=0.548$ \\
\hline $\begin{array}{l}\text { Revised MDS UPDRS } \\
\text { nonmotor aspects of } \\
\text { experiences of daily living }\end{array}$ & $7.4 \pm 5.1$ & $5.7 \pm 4.4$ & $t=-1.53, p=0.128$ \\
\hline $\begin{array}{l}\text { Revised MDS UPDRS motor } \\
\text { aspects of experiences of } \\
\text { daily living }\end{array}$ & $8.9 \pm 6.7$ & $8.1 \pm 5.7$ & $t=-0.58, p=0.562$ \\
\hline $\begin{array}{l}\text { Revised MDS UPDRS motor } \\
\text { examination score }\end{array}$ & $27.6 \pm 10.9$ & $25.1 \pm 11.2$ & $t=-0.93, p=0.35$ \\
\hline Hoehn and Yahr score & $2.3 \pm 0.44$ & $2.3 \pm 0.49$ & $t=-0.28, p=0.97$ \\
\hline
\end{tabular}

neocortical VOIs were defined using semiautomated threshold delineation of the cortical gray matter signal.

All image frames were spatially coregistered within subjects with a rigid-body transformation to reduce the effects of subject motion during the imaging session. These motion-corrected PET frames were spatially coregistered to the T1-weighted magnetic resonance using standard coregistration procedures in SPM8b implemented in Matlab 2010b (Mathworks, Natick, MA). Timeactivity curves for each VOI were generated from the spatially aligned PET frames. ${ }^{11} \mathrm{C}$-DASB SERT and ${ }^{11} \mathrm{C}$-DTBZ VMAT2 distribution volume ratio was then estimated by using the Logan plot graphical analysis method, ${ }^{45}$ with the time-activity curves as the input function and the inferior posterior cerebellum as reference tissue for ${ }^{11} \mathrm{C}-\mathrm{DASB}{ }^{40,46}$ and the neocortex as reference tissue for ${ }^{11} \mathrm{C}-\mathrm{DTBZ} .{ }^{45,47,48} \mathrm{~A}$ noninvasive kinetic analysis of the k3 hydrolysis rate (AChE activity) was performed using a direct estimation of $\mathrm{k} 3$, without use of an arterial input function, based on the shape of the tissue time-activity curve alone. ${ }^{49}$

Standard pooled variance $t$ or Satterthwaite's method of approximate $t$ tests was used for group comparisons (SAS version 9.1; SAS institute, Cary, NC). Chi-square testing was used to compare gender distribution between the 2 groups. Standard $t$ tests were used during a post hoc analysis to determine whether relative differences in cholinergic denervation found between subgroups were confounded by potential mimics of oneiric behavior.

\section{Results}

\section{Demographic and Clinical Variables}

Twenty-seven subjects (33.8\%) had RBD symptoms based on MSQ responses. No significant differences between groups were seen in mean age, duration of motor disease, MoCA scores, H-Y scale, or UPDRS motor experiences of daily living or motor examination (Table 1). RBD symptoms were more frequent among male than female patients with PD $(p<0.05)$. A nonsignificant trend toward higher nonmotor aspects of experiences of daily living scores was seen in the subjects with RBD symptoms compared to those without ( $p=$ 0.128).

Neuropsychological test performance in the 2 patient groups is listed in Table 2. Subjects with RBD symptoms showed significantly lower performance on the verbal learning test compared to those without $(t=$ 3.913, $p=0.0002$ ). No significant differences were seen between groups in attention, visuospatial function, or executive function cognitive domain $z$ scores.

\section{AChE and VMAT2 PET Measures}

Table 3 lists the mean neocortical, limbic cortical, and thalamic AChE hydrolysis rates and striatal VMAT2 binding levels in each patient group. Subjects reported to have RBD symptoms $(n=27)$ exhibited significantly decreased AChE hydrolysis rates compared to subjects without $(\mathrm{n}=53)$ in Neocortical (see Figure 1), thalamic, and limbic cortical regions. In contrast, no significant differences emerged between groups in striatal VMAT2 binding. Analysis of covariance was performed to evaluate group differences in AChE rates while controlling for the degree of nigrostriatal denervation. The addition of 
TABLE 2: Cognitive Testing Performance for PD Subjects with and without RBD Symptoms

\begin{tabular}{llll} 
Score & $\begin{array}{l}\text { PD Subjects with } \\
\text { RBD Symptoms }\end{array}$ & $\begin{array}{l}\text { PD Subjects without } \\
\text { RBD Symptoms }\end{array}$ & $\begin{array}{l}\text { Statistical } \\
\text { Significance }\end{array}$ \\
Verbal learning $z$ score & $-0.857 \pm 1.013$ & $0.038 \pm 0.945$ & $t=3.913, p=0.0002^{\mathrm{a}}$ \\
Attention $z$ score & $0.013 \pm 1.586$ & $0.091 \pm 1.916$ & $t=0.181, p=0.857$ \\
Visuospatial function $z$ score & $-0.222 \pm 1.494$ & $-0.211 \pm 1.086$ & $t=-0.036, p=0.971$ \\
Executive function $z$ score & $-0.326 \pm 1.027$ & $-0.222 \pm 1.129$ & $t=0.399, p=0.690$ \\
\hline a Statistically significant. & & & \\
\hline
\end{tabular}

striatal DTBZ as a covariate did not change significant AChE group effects for any of the PMP regions (neocortex, $F=13.2, p<.0001$; thalamus, $F=11.5, p<$ .0001 ; and limbic cortex $(F=6.5, p=0.002)$.

\section{Subgroup Analysis of Cerebral SERT Binding}

Analysis of raphe nucleus and striatal SERT binding in the subset of patients who underwent $\left[{ }^{11} \mathrm{C}\right] \mathrm{DASB}$ PET imaging $(\mathrm{n}=35)$ failed to show significant differences between groups with $(\mathrm{n}=11)$ or without $(\mathrm{n}=24)$ symptoms of RBD (see Table 3).

\section{Post Hoc Analysis}

We performed a post hoc analysis using other questionnaire data aimed at assessing for symptoms of RLS and PLMD that could be perceived as RBD mimics by sleep partners (Table 4). Twelve of 80 subjects reported mild or worse symptoms of RLS on the IRLS. There were no significant differences seen in neocortical (RLS group: $0.0228 \pm 0.0023$ vs non-RLS group: $0.0227 \pm 0.0030$; $t=0.169, p=0.868)$, limbic cortical (0.0411 \pm 0.0052 vs $0.0412 \pm 0.0057 ; t=-0.06, p=0.952)$, or thalamic $(0.0415 \pm 0.0042$ vs $0.0413 \pm 0.0012 ; t=$ $0.127, p=0.899)$ AChE activity. No significant differences were seen between these groups in California Verbal Learning Test (CVLT) performance $(Z=-0.205 \pm$ 0.986 vs $-0.598 \pm 1.37 ; t=1.194, p=0.236)$. Using the Parkinson's Disease Sleep Scale, we compared the quartile of subjects with the most severe RLS symptom scores on questions 4 and 10 to the remainder of the cohort and again found no significant differences in neocortical $(0.0232 \pm 0.0023$ vs $0.0226 \pm 0.0024 ; t=$ $-0.973, p=0.334)$, limbic cortical $(0.041 \pm 0.005$ vs $0.041 \pm 0.001 ; t=0.4473, p=0.656)$, or thalamic

TABLE 3: Mean \pm SD Neocortical, Limbic Cortical, and Thalamic AChE Hydrolysis Rates $\left(\mathrm{k} 3 ; \mathrm{min}^{-1}\right)$, and Striatal VMAT2 DVR in Patients with and without Symptoms of RBD

\begin{tabular}{|c|c|c|c|}
\hline PET Methods & $\begin{array}{l}\text { PD with RBD sx, } \\
n=29\end{array}$ & $\begin{array}{l}\text { PD without RBD sx, } \\
\mathrm{n}=55\end{array}$ & $\begin{array}{l}\text { Statistical } \\
\text { Significance }\end{array}$ \\
\hline Neocortical AChE k3 & $0.0213 \pm 0.0018$ & $0.0236 \pm 0.0022$ & $t=4.55, p<0.0001^{\mathrm{a}}$ \\
\hline Limbic cortical AChE k3 & $0.0388 \pm 0.0029$ & $0.0423 \pm 0.0058$ & $t_{\text {approx }}=2.85, p=0.0056^{\mathrm{a}}$ \\
\hline Thalamic AChE k3 & $0.0388 \pm 0.0025$ & $0.0427 \pm 0.0042$ & $t_{\text {approx }}=4.49, p<0.0001^{\mathrm{a}}$ \\
\hline Putamen DTBZ DVR & $1.7793 \pm 0.2266$ & $1.8206 \pm 0.2981$ & $t=0.63, p=0.53$ \\
\hline Caudate DTBZ DVR & $1.9689 \pm 0.3216$ & $2.0189 \pm 0.3911$ & $t=0.57, p=0.57$ \\
\hline Raphe nucleus DASB DVR & $\begin{array}{l}2.8361 \pm 0.3081 \\
(\mathrm{n}=11)\end{array}$ & $\begin{array}{l}2.8002 \pm 0.3623 \\
(\mathrm{n}=24)\end{array}$ & $t=-0.28, p=0.77$ \\
\hline Striatal DASB DVR & $\begin{array}{l}2.2771 \pm 0.1522 \\
(\mathrm{n}=11)\end{array}$ & $\begin{array}{l}2.3014 \pm 0.2061 \\
(\mathrm{n}=24)\end{array}$ & $t=0.35, p=0.72$ \\
\hline \multicolumn{4}{|c|}{$\begin{array}{l}\text { Mean } \pm \text { SD raphe and striatal SERT DVR in the subset of patients who underwent }\left[{ }^{11} \mathrm{C}\right] \mathrm{DASB} \text { SERT positron emission } \\
\text { tomography imaging }(\mathrm{n}=35) \text {. Student } t \text { values are presented with levels of significance. Satterthwaite's method of approximate } \\
t \text { tests }\left(t_{\text {approx }}\right) \text { was used for comparison of groups with unequal variances. } \\
\text { aCtatistically significant. } \\
\text { AChE = acetylcholinesterase; DASB = benzonitrile; DTBZ }=\left[{ }^{11} \mathrm{C}\right] \text { dihydrotetrabenazine; DVR }=\text { distribution volume ratio; } \\
\mathrm{PD}=\text { Parkinson disease; RBD }=\text { rapid eye movement sleep behavior disorder; SD = standard deviation; SERT = serotonin } \\
\text { transporter; sx = symptoms; PET = positron emission tomography. }\end{array}$} \\
\hline
\end{tabular}




\section{TABLE 4: Number of Subjects with and without Symptoms of RBD on the MSQ Who Also Noted Symptoms of Restless Leg Syndrome on Either the IRLS or the PDSS}

\begin{tabular}{|c|c|c|}
\hline $\begin{array}{l}\text { RBD sx } \\
\text { on MSQ }\end{array}$ & $\begin{array}{l}\text { Negative Response } \\
\text { to Both IRLS } \\
\text { and PDSS }\end{array}$ & $\begin{array}{l}\text { Positive Response } \\
\text { to Either IRLS } \\
\text { or PDSS }\end{array}$ \\
\hline Negative & 39 (48.75\%) & $14(17.5 \%)$ \\
\hline Positive & $16(20 \%)$ & $11(13.75 \%)$ \\
\hline \multicolumn{3}{|c|}{$\begin{array}{l}\text { IRLS = International Restless Leg Scale; MSQ = Mayo } \\
\text { Sleep Questionnaire; PDSS = Parkinson's Disease Sleep } \\
\text { Scale; RBD = rapid eye movement sleep behavior disorder; } \\
\text { sx = symptoms. }\end{array}$} \\
\hline
\end{tabular}

$(0.4101 \pm 0.0033$ vs $0.0416 \pm 0.0044 ; t=0.4473, p$ $=0.656) \mathrm{AChE}$ activity. There were no differences in CVLT performance as well $(Z=-0.403 \pm 1.23$ vs $-0.218 \pm 0.989 ; t=0.679, p=0.499)$.

\section{Discussion}

This imaging study of 80 nondemented PD subjects indicates that RBD symptoms are associated preferentially with degeneration of brain cholinergic systems in PD. We found no association between the degree of striatal dopaminergic denervation or the loss of striatal or raphe nucleus serotoninergic terminals and a history of RBD symptoms. The $\left[{ }^{11} \mathrm{C}\right] \mathrm{PMP}$ AChE imaging method assesses the integrity of the 2 major brain cholinergic projection systems in the brain: neocortical and limbic cortical $\left[{ }^{11} \mathrm{C}\right] \mathrm{PMP}$ retention measures basal forebrain corticipetal projection system integrity, whereas thalamic $\left[{ }^{11} \mathrm{C}\right] \mathrm{PMP}$ retention is an index of the integrity of the pedunculopontine nucleus (PPN)-lateral dorsal tegmental nucleus (LDTN) projection system. The preferential association of diminished central nervous system cholinergic terminals with a history of RBD symptoms in these subjects suggests that these cholinergic systems are important in the pathophysiology of RBD in PD.

The risk of developing a neurodegenerative disease among patients with idiopathic RBD is roughly between 40 and $65 \%$ at 10 years, with approximately half of these patients developing PD. ${ }^{50}$ Previous studies have shown a link between nigrostriatal dopaminergic denervation and RBD in alpha-synucleinopathies. ${ }^{51,52}$ Although striatal dopaminergic denervation may be associated with idiopathic RBD, ${ }^{52}$ Kim et al recently demonstrated that the severity of nocturnal motor symptoms in idiopathic RBD does not correlate with striatal dopaminergic terminal density as measured by dopamine transporter imaging. ${ }^{53}$ Nigrostriatal dopaminergic denervation is a near universal hallmark of PD; however, only a subset of $\mathrm{PD}$ patients develop RBD. Additional underlying factors are therefore likely to play key roles in the pathogenesis of RBD.

RBD is likely caused by neuronal degeneration or dysfunction in brain regions that regulate suppression of skeletal muscle tone during REM sleep. Members of this complex circuit include the cholinergic PPN and LDTN, the serotonergic raphe nucleus, and glutamatergic and monoaminergic projections from the parabrachial-precoeruleus regions and the locus coeruleus (LC). ${ }^{54}$ Our results provide support for the concept that degeneration or dysfunction in this region of the brainstem causes $\mathrm{RBD}$, in that diminished thalamic cholinergic innervation is consistent with PPN-LDTN degeneration. A small case series has reported that acetylcholinesterase inhibitors such as donepezil can ameliorate the symptoms of RBD, consistent with the hypothesis that degeneration within brainstem cholinergic nuclei may play a role in the pathogenesis of RBD. ${ }^{55}$ A recent postmortem study of patients with Lewy body diseases, however, found no differences in the severity of PPN neuronal loss regardless of RBD symptoms during life, suggesting that alpha synuclein-mediated pathology in the pontine tegmentum alone may not be sufficient to cause RBD in PD. ${ }^{56}$

The cholinergic magnocellular nuclei of the basal forebrain complex is affected by Lewy body pathology in Braak stage 3 and may account for some of the association between RBD and cognitive impairment in PD. ${ }^{6}$ Although the basal forebrain complex plays an essential role in cognition, it also has strong interconnections with the brainstem nuclei regulating sleep and atonia during REM. Recent experiments indicate that the basal forebrain complex may play an important role in the regulation of arousal and sleep. ${ }^{57}$ This arousal projection system of the basal forebrain complex is linked to the pontine parabrachial-precoeruleus complex, suggesting the existence of functionally important interactions between these pontine nuclei and basal forebrain cholinergic systems. ${ }^{57}$ What role the basal forebrain complex may play in the pathogenesis of idiopathic RBD is currently unclear, although early neuronal dysfunction in the cholinergic nucleus basalis of Meynert provides a potential mechanism for the link between antecedent idiopathic RBD and the subsequent development of both $\mathrm{PD}$ and dementia. ${ }^{58}$ Further in vivo studies of the integrity of the basal forebrain complex, the PPN, and the LC in idiopathic RBD may help to clarify this association.

Compared to nondemented subjects with PD, patients with Alzheimer dementia (AD) have more pronounced cholinergic denervation in limbic cortical regions with less severe denervation seen in the 
neocortex. ${ }^{8}$ Thalamic cholinergic denervation is not a recognized feature of $\mathrm{AD} .{ }^{59,60}$ Neocortical cholinergic denervation is more severe and extensive in parkinsonian dementia compared to $\mathrm{AD} .^{8}$ The degree of neocortical cholinergic denervation seen in our cohort of PD subjects with RBD symptoms is approximately intermediate between the levels seen in parkinsonian dementia and PD without dementia. We did not find significant differences in cognitive test performance between the groups with and without RBD symptoms except for significantly decreased performance on the verbal learning test in subjects with symptoms of RBD. These findings are consistent with a previous study by our group showing an association between hyposmia in $\mathrm{PD}$, poor performance on verbal learning testing, and neocortical and limbic cortical cholinergic denervation. ${ }^{32}$ We propose that the presence of basal forebrain cholinergic dysfunction may represent a pathological mechanism contributing to the development of dementia in patients who suffer from idiopathic RBD.

A limitation of our study is that the diagnosis of RBD is based on questionnaire data and not confirmed by polysomnography (PSG). Others have argued that the diagnostic criteria for probable RBD can be fulfilled by positive responses to questions listed on the MSQ supplemented by affirmation of specific subquestions, although the gold standard remains video PSG. ${ }^{61}$ The proportion of subjects with RBD symptoms in our cohort $(33.8 \%)$ is within the range of previously published estimates of RBD prevalence among patients with PD (15-47\%). ${ }^{62}$ However, bed partner-reported dream enactment behavior in some cases conceivably could reflect other common sleep phenomena in PD such as periodic limb movements of sleep, restless leg syndrome, or a manifestation of obstructive sleep apnea. Our post hoc analysis suggests that presence of RLS/PLMD is not associated with cholinergic denervation in our cohort. The MSQ is a well-validated instrument and may yield an increased sensitivity for detecting dream enactment behavior compared to traditional PSG methods. ${ }^{17}$ The relatively lower specificity (74\%) of this questionnaire relative to PSG-based diagnosis may also reflect the intermittent presence of increased motor tone during REM sleep, a finding that may not fully be captured by a single overnight PSG. Determining the presence of REM sleep without atonia on PSG is also not straightforward, and such analyses may be confounded by potentially poor inter-rater reliability. ${ }^{63}$ Furthermore, the false-positive rate of $26 \%$ seen in the MSQ validation study decreased in the study population to $16 \%$ with an affirmative answer to subquestion $1 \mathrm{~d}$ versus $17 \%$ with an affirmative answer to subquestion 1e. We required affirmative responses to both $1 \mathrm{~d}$ and $1 \mathrm{e}$ to characterize subjects as having RBD symptoms. It is also worth noting that the MSQ validation study examined subjects with $\mathrm{AD}$, mild cognitive impairment, and tauopathies, as well as subjects without any known neurodegenerative disorders. With the higher prevalence of RBD in synucleinopathies, we expect a higher specificity of the MSQ in this population. Our study cohort represents a highly enriched PD sample, as clinical diagnosis was confirmed by nigrostriatal denervation on DTBZ PET imaging.

An additional point worth noting is that we are interpreting decreased PMP retention as evidence of cholinergic terminal degeneration, although we cannot exclude the possibility of an alteration in AChE expression. ${ }^{64}$ The relationship between cholinergic denervation and RBD symptoms in this study represents a strong correlation, although it does not represent proof of causation. For example, subjects with RBD symptoms in our cohort may have underlying brain changes consistent with a predementia state, which conceivably could confer a stronger association with symptoms of RBD than does the cholinergic deficit that is associated with such a predementia state. We only studied SERT binding in a subset of subjects, so we cannot exclude a small difference in serotoninergic raphe neuron integrity between subjects with and without RBD symptoms. The essentially equivalent mean regional DASB binding values of these groups indicate that if such a difference exists, it would be rather small.

Despite these limitations, our data suggest that cholinergic projection systems in the pontine tegmentum and basal forebrain complex could play a key role in the pathogenesis of RBD in PD and may contribute to the propensity to develop cognitive impairment found in such patients. Differential cholinergic systems degeneration in PD may reflect the existence of specific PD subtypes. We speculate that cholinergic basal forebrain complex dysfunction may have similar effects on sleep and cognitive function in a number of related neurodegenerative diseases. RBD and PD likely represent overlapping neurodegenerative phenomena with common neuroanatomical substrates. Changes in the cholinergic nervous systems within the brainstem and basal forebrain may play a vital role in the pathophysiology of RBD and hence have the potential to offer insight into the underlying heterogeneity of a number of neurodegenerative diseases. Longitudinal observational studies that follow risk factors, clinical features, their association with in vivo pathology involving various neuronal systems, and their correlation with postmortem histopathologic findings are necessary to further investigate the cause of RBD in $\mathrm{PD}$ and in other neurodegenerative diseases. 


\section{Acknowledgments}

Supported by the Michael J. Fox Foundation, the Department of Veterans Affairs, and NIH grants P01 NS015655: Kirk Frey (KAF) and R01 NS070856: Nicolaas Bohnen (NIB).

We thank C. Minderovic, V. Rogers, and the PET technologists, cyclotron operators, and chemists for their assistance, and the editors and anonymous reviewers for constructive comments.

\section{Potential Conflicts of Interest}

R.D.C.: board membership, American Academy of Sleep Medicine, American Sleep Medicine Foundation, American Board of Sleep Medicine, International Pediatric Sleep Association, Pavad Medical (received stock options; company is now defunct), Sweet Dreamzzz (not for profit company), Sleep Research Society; consultancy, Arena Pharmaceuticals, Guidepoint Global, OrbiMed Advisors; grants/grants pending, NIH, University of Michigan Medical School, University of Michigan Medical Innovation Center; patents, University of Michigan holds patents and patents pending for signal analysis algorithms relevant to diagnosis of sleep disorders and device to treat obstructive sleep apnea (author could conceivably receive monetary benefit in the future); royalties, Section Editor of UpToDate; gifts received by University of Michigan from Philips Respironics and Fisher Paykel at author's request for educational purposes. K.A.F.: consultancy, AVID Pharmaceuticals, Bayer Schering Pharmaceuticals, MIMvista; grants/grants pending, General Electric; stock/stock options, General Electric. NIB: grants/grants pending, NIH.

\section{References}

1. Schenck C, Bundlie S, Ettinger M, Mahowald M. Chronic behavioral disorders of human REM sleep: a new category of parasomnia. Sleep 1986;9:293-308.

2. Gagnon JF, Vendette M, Postuma RB, et al. Mild cognitive impairment in rapid eye movement sleep behavior disorder and Parkinson's disease. Ann Neurol 2009:66:39-47.

3. Marion MH, Qurashi M, Marshall G, Foster O. Is REM sleep behaviour disorder (RBD) a risk factor of dementia in idiopathic Parkinson's disease? J Neurol 2008;255:192-196.

4. Williams-Gray CH, Evans JR, Goris A, et al. The distinct cognitive syndromes of Parkinson's disease: 5 year follow-up of the CamPaIGN cohort. Brain 2009;132(pt 11):2958-2969.

5. Kehagia AA, Barker RA, Robbins TW. Neuropsychological and clinical heterogeneity of cognitive impairment and dementia in patients with Parkinson's disease. Lancet Neurol 2010;9: $1200-1213$

6. Braak H, Del Tredici K, Rüb U, et al. Staging of brain pathology related to sporadic Parkinson's disease. Neurobiol Aging 2003;24:197-211.

7. Ruberg M, Rieger F, Villageois A, et al. Acetylcholinesterase and butyrylcholinesterase in frontal cortex and cerebrospinal fluid of demented and non-demented patients with Parkinson's disease. Brain Res 1986;362:83-91.

8. Bohnen NI, Kaufer DI, Ivanco LS, et al. Cortical cholinergic function is more severely affected in parkinsonian dementia than in Alzheimer disease: an in vivo positron emission tomographic study. Arch Neurol 2003;60:1745-1748.

9. Hilker R, Thomas AV, Klein JC, et al. Dementia in Parkinson disease: functional imaging of cholinergic and dopaminergic pathways. Neurology 2005;65:1716-1722.

10. Shimada H, Hirano S, Shinotoh H, et al. Mapping of brain acetylcholinesterase alterations in Lewy body disease by PET. Neurology 2009;73:273-278.

11. Fox SH, Chuang R, Brotchie JM. Serotonin and Parkinson's disease: on movement, mood, and madness. Mov Disord 2009:24: 1255-1266

12. Albin RL, Koeppe RA, Bohnen NI, et al. Spared caudal brainstem SERT binding in early Parkinson's disease. J Cereb Blood Flow Metab 2008;28:441-444

13. Hughes AJ, Daniel SE, Kilford L, Lees AJ. Accuracy of clinical diagnosis of idiopathic Parkinson's disease: a clinicopathologic study of 100 cases. J Neurol Neurosurg Psychiatry 1992;55: $181-184$

14. Hoehn M, Yahr M. Parkinsonism: onset, progression, and mortality. Neurology 1967;17:427-442

15. Nasreddine ZS, Phillips NA, Bedirian V, et al. The Montreal Cognitive Assessment, MoCA: a brief screening tool for mild cognitive impairment. J Am Geriatr Soc 2005;53:695-699.

16. Goetz CG, Fahn S, Martinez-Martin P, et al. Movement Disorder Society-sponsored revision of the Unified Parkinson's Disease Rating Scale (MDS-UPDRS): process, format, and clinimetric testing plan. Mov Disord 2007;22:41-47.

17. Boeve BF, Molano JR, Ferman TJ, et al. Validation of the Mayo Sleep Questionnaire to screen for REM sleep behavior disorder in an aging and dementia cohort. Sleep Med 2011; 12:445-453.

18. Abetz L, Arbuckle R, Allen RP, et al. The reliability, validity and responsiveness of the International Restless Legs Syndrome Study Group rating scale and subscales in a clinical-trial setting. Sleep Med 2006;7:340-349.

19. Chaudhuri KR, Martinez-Martin P. Clinical assessment of nocturnal disability in Parkinson's disease: the Parkinson's Disease Sleep Scale. Neurology 2004;63(8 suppl 3):S17-S20.

20. Gomez-Esteban JC, Zarranz JJ, Tijero B, et al. Restless legs syndrome in Parkinson's disease. Mov Disord 2007;22:1912-1916.

21. Nomura T, Inoue Y, Nakashima K. Clinical characteristics of Restless legs syndrome in patients with Parkinson's disease. J Neurol Sci 2006:250:39-44.

22. Aarsland D, Bronnick K, Larsen JP, et al. Cognitive impairment in incident, untreated Parkinson disease: the Norwegian ParkWest study. Neurology 2009;72:1121-1126.

23. Bronnick K, Alves G, Aarsland D, et al. Verbal memory in drug-naive, newly diagnosed Parkinson's disease. The retrieval deficit hypothesis revisited. Neuropsychology 2011;25:114-124.

24. Delis DC, Kramer JH, Kaplan E, Ober BA. California Verbal Learning Test manual, adult version. 2nd ed. San Antonio, TX: Psychological Corporation, 2000.

25. Beatty WW, Monson N. Picture and motor sequencing in Parkinson's disease. J Geriatr Psychiatry Neurol 1990;3:192-197.

26. Wechsler D. WAIS III technical manual. San Antonio, TX: Psychological Corporation, 1997.

27. Delis DC, Kaplan E, Kramer JH. Delis-Kaplan Executive Function System (D-KEFS): examiner's manual. San Antonio, TX: Psychological Corporation, 2001. 
28. Schmitt FA, Farlow MR, Meng $X$, et al. Efficacy of rivastigmine on executive function in patients with Parkinson's disease dementia. CNS Neurosci Ther 2010;16:330-336.

29. Stroop JR. Studies of interference in serial verbal reactions. J Exp Psychol 1935;18:643-662.

30. Bohnen NI, Jolles J, Twijnstra A. Modification of the Stroop Color Word Test improves differentiation between patients with mild head injury and matched controls. Clin Neuropsychol 1992;6:178-184.

31. Woodward TS, Bub DN, Hunter MA. Task switching deficits associated with Parkinson's disease reflect depleted attentional resources. Neuropsychologia 2002;40:1948-1955.

32. Bohnen NI, Muller ML, Kotagal V, et al. Olfactory dysfunction, central cholinergic integrity and cognitive impairment in Parkinson's disease. Brain 2010;133(pt 6):1747-1754.

33. Galtier I, Nieto A, Barroso J, Norelis Lorenzo J. Visuospatial learning impairment in Parkinson disease [in Spanish]. Psicothema 2009;21:21-26.

34. Benton AL, Varney NR, Hamsher K. Judgment of line orientation, form V. lowa City, IA: University of lowa Hospitals, 1975.

35. Vander Borght TM, Sima AA, Kilbourn MR, et al. [3H]methoxytetrabenazine: a high specific activity ligand for estimating monoaminergic neuronal integrity. Neuroscience 1995;68:955-962.

36. Wilson JM, Levey Al, Rajput A, et al. Differential changes in neurochemical markers of striatal dopamine nerve terminals in idiopathic Parkinson's disease. Neurology 1996;47:718-726.

37. Frey KA, Koeppe RA, Kilbourn MR. Imaging the vesicular monoamine transporter. Adv Neurol 2001;86:237-247.

38. Bohnen NI, Albin RL. The cholinergic system and Parkinson disease. Behav Brain Res 2011;221:564-573.

39. Shute CC, Lewis PR. Electron microscopy of cholinergic terminals and acetylcholinesterase-containing neurones in the hippocampal formation of the rat. Z Zellforsch Mikrosk Anat 1966;69:334-343.

40. Meyer J. Imaging the serotonin transporter during major depressive disorder and antidepressant treatment. J Psychiatry Neurosci 2007;32:86-102.

41. Thompson CJ, Kecani S, Boelen S. Evaluation of a neck-shield for use during neurological studies with a whole-body PET scanner. IEEE Trans Nucl Sci 2001;48:1512-1517.

42. Jewett DM, Kilbourn MR, Lee LC. A simple synthesis of [11C]dihydrotetrabenazine (DTBZ). Nucl Med Biol 1997;24:197-199.

43. Innis RB, Cunningham VJ, Delforge J, et al. Consensus nomenclature for in vivo imaging of reversibly binding radioligands. J Cereb Blood Flow Metab 2007;27:1533-1539.

44. Snyder SE, Tluczek L, Jewett DM, et al. Synthesis of 1-[11C]methylpiperidin-4-yl propionate ([11C]PMP) for in vivo measurements of acetylcholinesterase activity. Nucl Med Biol 1998;25:751-754.

45. Logan J, Fowler JS, Volkow ND, et al. Distribution volume ratios without blood sampling from graphical analysis of PET data. J Cereb Blood Flow Metab 1996;16:834-840.

46. Ginovart N, Wilson A, Meyer J, et al. Positron emission tomography quantification of [(11)C]-DASB binding to the human serotonin transporter: modeling strategies. J Cereb Blood Flow Metab $2001 ; 21: 1342-1353$.

47. Koeppe RA, Frey KA, Kume A, et al. Equilibrium versus compartmental analysis for assessment of the vesicular monoamine transporter using (+)-alpha-[11C]dihydrotetrabenazine (DTBZ) and positron emission tomography. J Cereb Blood Flow Metab 1997; 17:919-931.

48. Koeppe RA, Frey KA, Kuhl DE, Kilbourn MR. Assessment of extrastriatal vesicular monoamine transporter binding site density using stereoisomers of [11C]dihydrotetrabenazine. J Cereb Blood Flow Metab 1999;19:1376-1384

49. Koeppe RA, Frey KA, Snyder SE, et al. Kinetic modeling of N[11C]methylpiperidin-4-yl propionate: alternatives for analysis of an irreversible positron emission tomography tracer for measurement of acetylcholinesterase activity in human brain. J Cereb Blood Flow Metab 1999;19:1150-1163.

50. Postuma RB, Gagnon JF, Montplaisir J. Clinical prediction of Parkinson's disease: planning for the age of neuroprotection. J Neurol Neurosurg Psychiatry 2010;81:1008-1013.

51. Gilman S, Koeppe R, Chervin R, et al. REM sleep behavior disorder is related to striatal monoaminergic deficit in MSA. Neurology 2003;8:29-34.

52. Albin R, Koeppe R, Chervin R. Decreased striatal dopaminergic innervation in REM sleep behavior disorder. Neurology 2000;55: 1410-1412.

53. Kim YK, Yoon IY, Kim JM, et al. The implication of nigrostriatal dopaminergic degeneration in the pathogenesis of REM sleep behavior disorder. Eur J Neurol 2010;17:487-492.

54. Boeve B, Silber M, Saper C, et al. Pathophysiology of REM sleep behaviour disorder and relevance to neurodegenerative disease. Brain 2007;130(pt 11):2770-2788.

55. Ringman JM, Simmons JH. Treatment of REM sleep behavior disorder with donepezil: a report of three cases. Neurology 2000;55: 870-871.

56. Dugger BN, Murray ME, Boeve BF, et al. Neuropathological analysis of brainstem cholinergic and catecholaminergic nuclei in relation to REM sleep behaviour disorder. Neuropathol Appl Neurobiol 2011 Jun 23 [Epub ahead of print].

57. Fuller P, Sherman D, Pedersen NP, et al. Reassessment of the structural basis of the ascending arousal system. J Comp Neurol 2011;519:933-956.

58. Postuma RB, Gagnon JF, Vendette M, et al. Quantifying the risk of neurodegenerative disease in idiopathic REM sleep behavior disorder. Neurology 2009;72:1296-1300.

59. Kuhl DE, Koeppe RA, Minoshima S, et al. In vivo mapping of cerebral acetylcholinesterase activity in aging and Alzheimer's disease. Neurology 1999;52:691-699

60. Mesulam M. The cholinergic lesion of Alzheimer's disease: pivotal factor or side show? Learn Mem 2004;11:43-49.

61. Boeve BF. REM sleep behavior disorder: updated review of the core features, the REM sleep behavior disorder-neurodegenerative disease association, evolving concepts, controversies, and future directions. Ann N Y Acad Sci 2010;1184:15-54.

62. Gagnon JF, Bedard MA, Fantini ML, et al. REM sleep behavior disorder and REM sleep without atonia in Parkinson's disease. Neurology 2002;59:585-589.

63. Bliwise DL. Challenges in the assessment of dream enactment behavior. Sleep Med 2011;12:429-430.

64. Bohnen NI, Frey KA. Imaging of cholinergic and monoaminergic neurochemical changes in neurodegenerative disorders. Mol Imaging Biol 2007;9:243-257. 